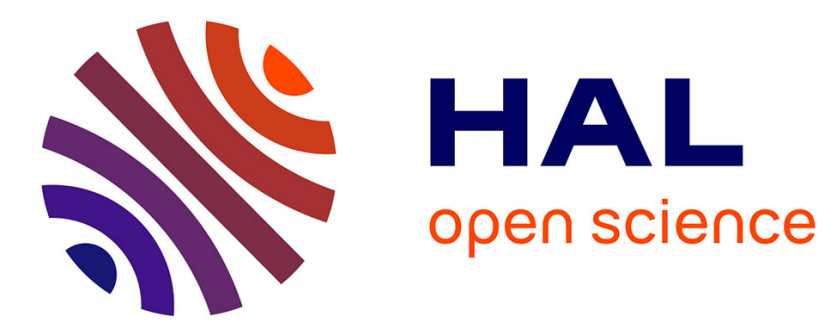

\title{
Nonlinear Effects of Sound Waves in High-Tc-Superconductors
}

\author{
A. Geerkens, S. Ewert
}

\section{To cite this version:}

A. Geerkens, S. Ewert. Nonlinear Effects of Sound Waves in High-Tc-Superconductors. Journal de Physique IV Proceedings, 1996, 06 (C8), pp.C8-465-C8-468. 10.1051/jp4:19968100 . jpa-00254528

\section{HAL Id: jpa-00254528 https://hal.science/jpa-00254528}

Submitted on 1 Jan 1996

HAL is a multi-disciplinary open access archive for the deposit and dissemination of scientific research documents, whether they are published or not. The documents may come from teaching and research institutions in France or abroad, or from public or private research centers.
L'archive ouverte pluridisciplinaire HAL, est destinée au dépôt et à la diffusion de documents scientifiques de niveau recherche, publiés ou non, émanant des établissements d'enseignement et de recherche français ou étrangers, des laboratoires publics ou privés. 


\title{
Nonlinear Effects of Sound Waves in High- $T_{\mathrm{c}}$-Superconductors
}

\author{
A. Geerkens and S. Ewert \\ Technical University of Cottbus, Lehrstuhl für Experimentalphysik, P.O. Box 101344, 03013 Cottbus, \\ Germany
}

\begin{abstract}
High- $\mathrm{T}_{c}$-superconductors are studied by measuring the ultrasonic attenuation and the relative change of the sound velocity in various applied magnetic fields. High sound amplitudes reveal nonlinear effects of the fluxline lattice. Due to the coupling of the flux lines to the sound waves, the occurring effects show the pinning behaviour of a high- $\mathrm{T}_{\mathrm{c}}$-superconductor. Especially the results for a textured ceramic $\mathrm{Bi}_{1.6} \mathrm{~Pb}_{0.4} \mathrm{Sr}_{2} \mathrm{Ca}_{2} \mathrm{Cu}_{3} \mathrm{O}_{\mathrm{y}}$-superconductor are presented and discussed.
\end{abstract}

\section{INTRODUCTION}

The flux-line lattice in the mixed state of high- $T_{c}$-superconductors was investigated by many methods on bulk materials and thin films. Using ultrasonic measurements [1] the flux lines are mechanically excited instead of the mostly applied magnetic force for example measurements of the a.c. susceptibility. Similar to these results in ultrasonic measurements nonlinear effects appear, if the sound amplitude is high enough.

\section{EXPERIMENTAL}

The $\mathrm{Bi}_{1.6} \mathrm{~Pb}_{0.4} \mathrm{Sr}_{2} \mathrm{Ca}_{2} \mathrm{Cu}_{3} \mathrm{O}_{\mathrm{y}}$ (Bi-2223)-superconducting sample [2] was prepared by a sol-gel process [3]. The sample had $80 \%$ of the theoretical density due to ist porousity. The superconducting transition temperature $T_{c}=103 \mathrm{~K}$ was determined by measuring the a.c. susceptibility. The ultrasonic attenuation $\alpha$ was measured by using a pulse-echo technique. The relative change of the sound velocity $\Delta v / v$ was determined by a phase comparison method. The applied magnetic field was up to 8 Tesla. The sound waves were generated by $\mathrm{LiNbO}_{3}$ transducers with a resonance frequency of $10 \mathrm{MHz}$. These transducers provide a proportionality between the sound amplitude and the applied electrical voltage for a wide amplitude range. Therefore in the following discussion we identify the sound amplitude with the effective electrical voltage. The sound amplitude was varied between $1 \mathrm{~V}$ and $600 \mathrm{~V}$. To avoid warming up of the sample the pulse repetition rate was chosen small enough. The measurements were made for different orientations between magnetic field, sound wave direction and crystal direction. Once we used a transverse sound wave, where the amplitude $\underline{u}$ is perpendicular to the wave vector $\underline{k}$. The $\underline{c}$-axis of the textured sample was parallel to the applied magnetic field B. In the second investigated geometry a hard mode was activated by a longitudinal transducer ( $\underline{\mathrm{u}} \mid \mathrm{I} \underline{\mathrm{k}}$ ) with $\underline{\mathrm{k}} \| \underline{\mathrm{c}}$ and the magnetic field perpendicular to the $\underline{\mathrm{c}}$-axis of the $\mathrm{Bi}-2223$ superconductor.

\section{RESULTS AND DISCUSSION}

\subsection{Ultrasonic attenuation}

\subsubsection{Magnetic field dependence}

The magnetoacoustic measurements show the elastic constants of the flux-line lattice. The following results were taken for the $\underline{\mathrm{c}} \mathrm{II} \mathrm{B}$ geometry and the hard mode. The measurements were performed field cooled and with low sound amplitudes. The flux-lines have attained their equilibrium position and ultra- 


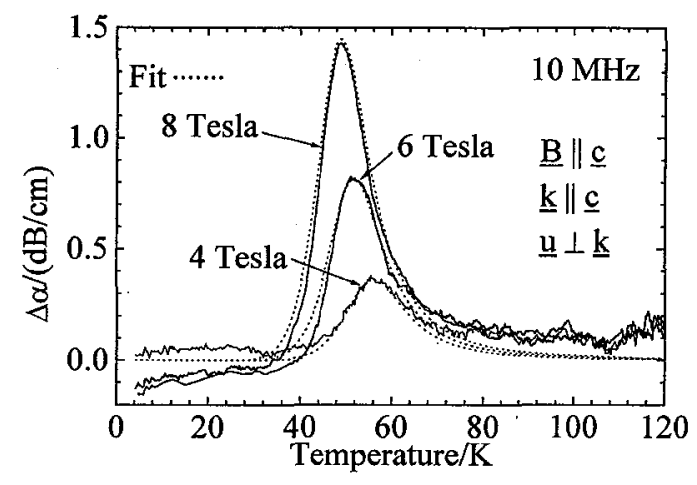

Figure 1: Temperature dependence of ultrasonic attenuation for a transverse sound mode with $\underline{\mathrm{k}} \| \mathrm{c}$ and $\underline{\mathrm{c}} \| \mathrm{B}$ in $\mathrm{Bi}-2223$ for various magnetic fields.

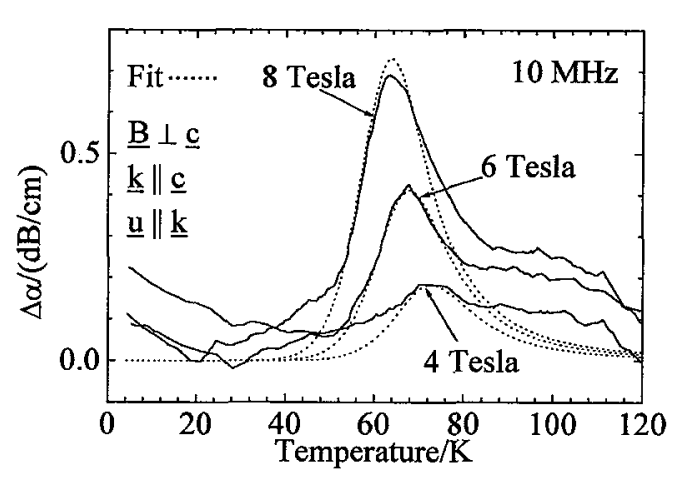

Figure 2: Temperature dependence of ultrasonic attenuation for a longitudinal sound mode with $\underline{\mathrm{k}} \| \underline{\mathrm{c}}$ and $\underline{\mathrm{c}} \perp \underline{\mathrm{B}}$ (hard mode) in Bi-2223 for various magnetic fields.

sound waves will move them away from equilibrium. After that they relax into equilibrium again. The temperature dependence of the ultrasonic attenuation $\Delta \alpha=\alpha(B)-\alpha(0)$ was measured for different magnetic fields as shown in Figs. 1 and 2. The attenuation obtains a maximum at $T_{\text {dep }}$ below $T_{c}=103 \mathrm{~K}$ depending on the magnetic field and the sound mode. This temperature can be explained as crossover from a depinned $\left(T>T_{d e p}\right)$ to a pinned $\left(T<T_{d e p}\right)$ state of the flux-lines. The effect increases proportional to $\mathrm{B}^{2}$. For higher magnetic fields the flux-line density is higher, so the depinning temperature decreases. At 8 Tesla $\mathrm{T}_{\text {dep }}$ reaches $64 \mathrm{~K}$ for the hard mode $(\underline{c} \perp \underline{B})$, but only $49 \mathrm{~K}$ for the $\underline{\mathbf{c}}$ II $\underline{\mathrm{B}}$ geometry. This reveals the different behaviour of the sample depending on the activated modes. These magnetoacoustic effects are explained by a theoretical model $[4,5,1]$ in the framework of thermally assisted flux flow and in previous experimental results $[6,7,8]$. For example for the $\underline{\mathrm{c}}$ II $\underline{\mathrm{B}}$ measurements we get

(1) $\Delta \alpha=\frac{\omega^{2}}{2 \rho_{m} \mathrm{v}_{\mathrm{t}}^{3}} \frac{\mathrm{C}_{44}{ }^{2} \Gamma \mathrm{k}^{2}}{\omega^{2}+\left(\mathrm{C}_{44} \Gamma \mathrm{k}^{2}\right)^{2}}$ and (2) $\frac{\Delta \mathrm{v}}{\mathrm{v}}(\mathrm{B})-\frac{\Delta \mathrm{v}}{\mathrm{v}}(0)=\frac{1}{2 \rho_{m} \mathrm{v}_{\mathrm{t}}^{2}} \frac{\mathrm{C}_{44} \omega^{2}}{\omega^{2}+\left(\mathrm{C}_{44} \Gamma \mathrm{k}^{2}\right)^{2}}$ with

$\omega / 2 \pi$ being the frequency, $v_{t}=1830 \mathrm{~m} / \mathrm{s}$ the transverse sound velocity for this orientation and $\rho_{\mathrm{m}}$ the density of the sample. The tilt modulus of the flux-line lattice is $\mathrm{C}_{44}=\mathrm{B}^{2} / \mu_{\mathrm{o}}$. The relaxation coefficient $\Gamma$ can be determined by

(3) $\Gamma=\rho / B^{2}=\left(\rho_{n} / B^{2}\right) \exp [-U / T]$ with $\rho$ the dc resistivity.

$\mathrm{U}$ is the activation energy and $\rho_{\mathrm{n}}$ a constant which can be set $\rho_{\mathrm{n}}=0.1 \Omega \mathrm{cm}$ for the Bi-2223 superconductor [9]. Inserting the expression (3) in (1) and (2) we get an attenuation peak at

(4) $T_{\text {dep }}=U / \ln \left(\rho_{n} k^{2} / \omega \mu_{\mathrm{o}}\right)$ and a step in velocity at the same temperature.

With $\rho_{\mathrm{n}}$ and $U$ fixed we are left with no adjustable parameter. The resulting theoretical prediction is presented by the fit curves in Fig. 1. They show a good agreement between theory and experiment not only in the position, but also in the shape of the attenuation peak. The investigated longitudinal hard mode corresponds to the compression modulus $\mathrm{C}_{11}$ of the flux-line lattice. But for the used long wavelengths $C_{11}$ can be set equal to $C_{44}$ [8]. So the same equations can be used with the longitudinal sound velocity of $v_{1}=2220 \mathrm{~m} / \mathrm{s}$. These fits shown in Fig. 2 reflect as well the behaviour of this sound mode. We conclude from these results that for small driving forces, flux-line motion is mainly diffusive in character [1].

\subsubsection{Amplitude Dependence}

Besides the dependence of the attenuation on the magnetic field, the sound amplitude affects the attenuation. Figs. 3 and 4 show $\Delta \alpha(T)$ for different sound amplitudes at a magnetic field of 8 Tesla. The attenuation maximum does not change for small sound amplitudes [6]. But for higher amplitudes $T_{\max }$ is shifted to lower temperatures and finally, if the amplitude is further increased the height of the maximum decreases, so the effects get smaller. We interpret the crossover point between linear and nonlinear behav- 


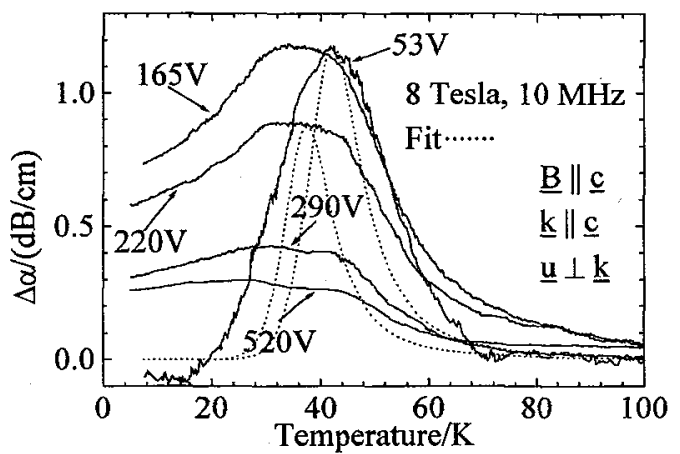

Figure 3: Temperature dependence of ultrasonic attenuation ( $\underline{\mathrm{u}} \perp \underline{\mathrm{k}}, \underline{\mathrm{k}} \mathrm{Il} \underline{\mathrm{c}}$ and $\underline{\mathrm{c}} \| \underline{\mathrm{B}}$ ) in $\mathrm{Bi}-2223$ for various sound amplitudes.

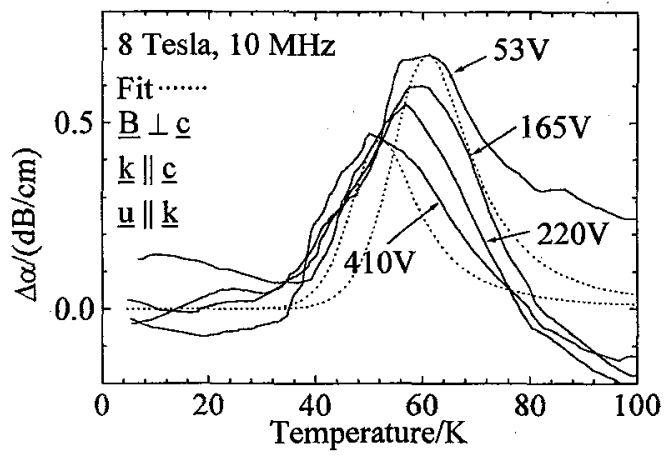

Figure 4: Temperature dependence of ultrasonic attenuation ( $\underline{\mathbf{u}}\|\underline{\mathrm{k}}, \underline{\mathrm{k}}\| \mathrm{l} \underline{\mathrm{c}}$ and $\underline{\mathrm{c}} \perp \underline{\mathrm{B}}$ ) in $\mathrm{Bi}-2223$ for various sound amplitudes.

iour as thermally activated flux motion due to the applied high amplitude [7]. Besides higher temperatures of the attenuation maxima for the same applied voltage for the $\underline{\mathrm{c}} \perp \underline{\mathrm{B}}$ geometry (hard mode), the attenuation peaks are broader for $\underline{\mathrm{c}}$ II $\underline{\mathrm{B}}$. This also reveals the easier motion of the flux-lines for $\underline{\mathrm{c}}$ II $\underline{\mathrm{B}}$. The fits show the correct position of the attenuation peak for a sound amplitude-dependent activation energy. But the used theory does not consider the broadening and the decrease of the effect.

\subsection{Sound velocity}

Besides measurements of the ultrasonic attenuation the relative change of the sound velocity $\Delta \mathrm{v} / \mathrm{v}$ was investigated. The temperature dependence of $\Delta v / v$ for a high and a low sound amplitude is shown in Figs. 5 and 6 . For each sound amplitude a field cooled measurement of $\Delta v / v$ at 8 Tesla and the corresponding result at 0 Tesla are compared. These results reveal the elastic constant of the flux-line lattice as a step in the sound velocity below the pinning transition [10]. This step in $\Delta v / v$ corresponds to the temperature of the attenuation maximum. For $\underline{c}$ II $\underline{B}$ the difference between the step temperature for the here shown low and high amplitudes is $7 \mathrm{~K}$, while for $\underline{\mathrm{c}} \perp \underline{\mathrm{B}}$ it is $12 \mathrm{~K}$. The decrease of the depinning temperature with increasing the amplitude is stronger for the hard mode. So these nonlinear effects are affected by the anisotropy of the pinning potentials [8]. The step magnitude at $4.2 \mathrm{~K}$ increases with increasing magnetic field, but decreases with increasing sound amplitude, especially for $\underline{\mathbf{c}}$ II $\underline{\mathrm{B}}$.

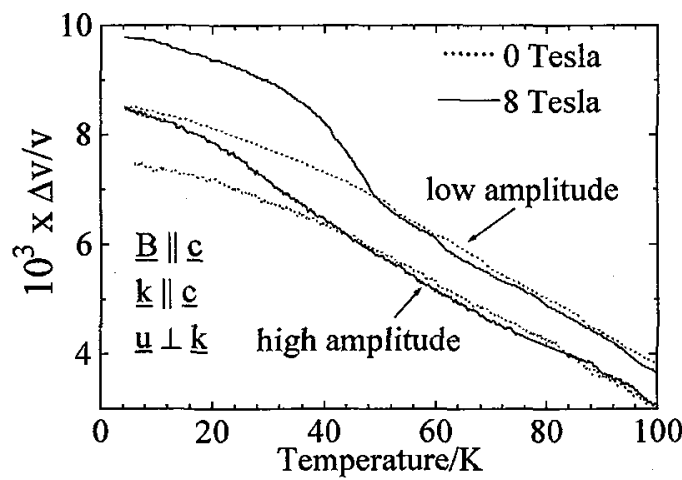

Figure 5: Temperature dependence of relative sound velocity $\Delta v / v$ in $\mathrm{Bi}-2223$ for various sound amplitudes at 8 Tesla and 0 Tesla for $\underline{\mathrm{c}} \| \mathrm{B}$.

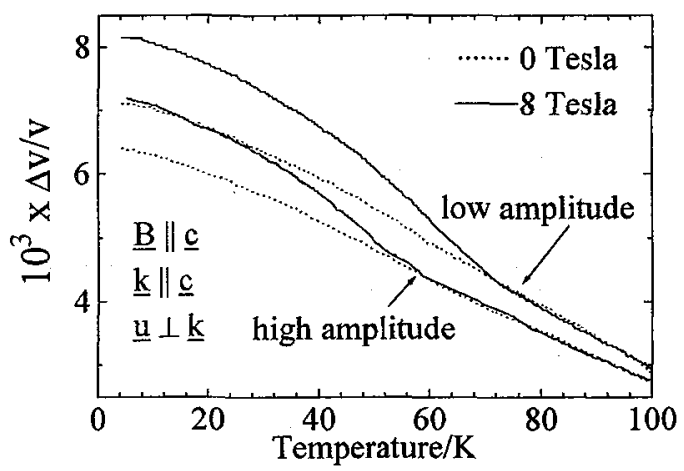

Figure 6: Temperature dependence of relative sound velocity $\Delta v / v$ in $\mathrm{Bi}-2223$ for various sound amplitudes at 8 Tesla and 0 Tesla for the hard mode. 


\subsection{Activation energy}

In Figs. 7 and 8 the activation energies $U$ for different sound amplitudes in various magnetic fields are presented. This energy is necessary to move the pinned flux-lines away from their pinning potentials. The activation energy was determined by equation (3). The decrease of $U$ with increasing sound amplitudes reflects the nonlinear behaviour of the $\mathrm{Bi}-2223$ superconductor. This corresponds to the shift of the attenuation peak for high amplitudes. We get a linear decrease of the activation energy with increasing sound amplitude. Additionally the activation energy decreases with higher magnetic fields. The clearly higher activation energies for the hard mode can be observed due to the harder movement of the flux-lines between different ab-planes of the strongly anisotropic Bi-2223 superconductor. From a certain high amplitude of the hard mode the activation energy increases with increasing amplitude. For very high amplitudes the flux-lines must be moved to places, which are occupied by other flux-lines. For this depinning of the flux-lines high energies are necessary. This behaviour starts at lower amplitudes for higher magnetic fields due to the higher density of the flux-lines.

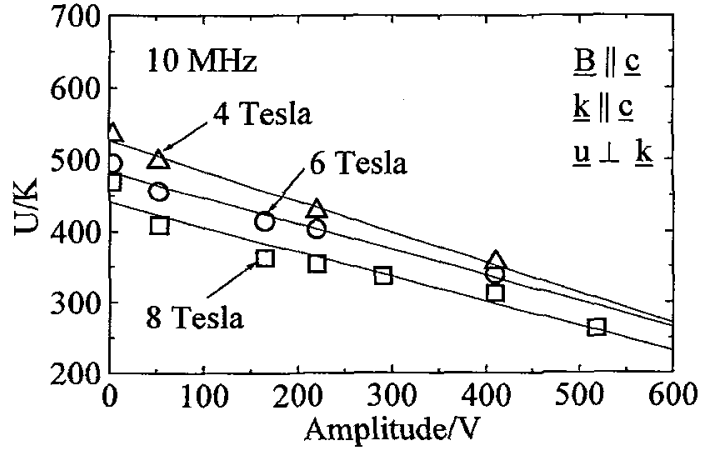

Figure 7: Activation energy versus sound amplitude for $\mathrm{Bi}-2223$ in different magnetic fields for $\underline{\mathrm{c}} \| \underline{\mathrm{B}}$.

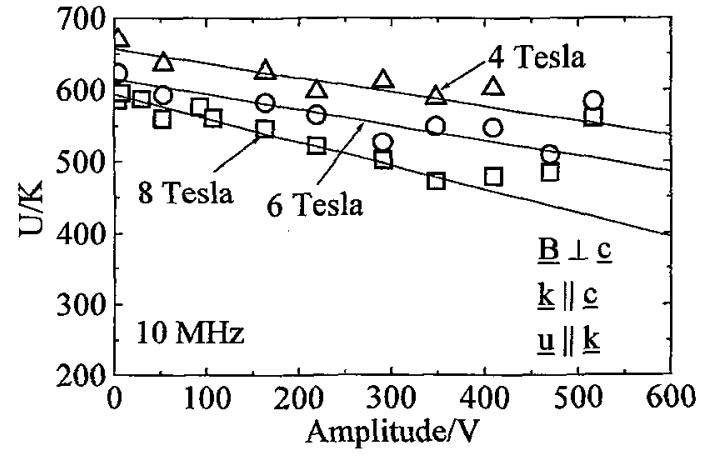

Figure 8: Activation energy versus sound amplitude for $\mathrm{Bi}-2223$ in different magnetic fields for $\underline{\mathrm{c}} \perp \underline{\mathrm{B}}$ (hard mode).

\section{Acknowledgements}

We would like to thank Prof. Dr. W. Sander from the $2^{\text {nd }}$ Physical Institute, RWTH Aachen, and Dr. A. Comberg and Dr. J. Pankert from the Philips Research Laboratories, Aachen for interesting discussions.

\section{References}

[1] Pankert J., Marbach G., Comberg A., Lemmens P., Fröning P., and Ewert S., Phys. Rev. Lett. 65 (1990) 3052.

[2] $\mathrm{Bi}_{1.6} \mathrm{~Pb}_{0.4} \mathrm{Sr}_{2} \mathrm{Ca}_{2} \mathrm{Cu}_{3} \mathrm{O}_{\mathrm{y}}$ - sample prepared at the Philips Research Laboratories Aachen, Germany.

[3] Marbach G., de Vries J.W.C., Klee M., Passing H., and Stotz S., Studies of High Temperature Superconductors Vol.6, Ed. A. V. Narlikar (Nova Science Commack, NY 1990).

[4] Pankert J., Physica C 168 (1990) 335.

[5] Pankert J., Physica B 165-166 (1990) 1273.

[6] Pankert J., Comberg A., Lemmens P., Fröning P., and Ewert S., Physica C 182 (1991) 291.

[7] Lemmens P., Ewert S., and Pankert J., Physica C 185-189, (1991) 2271.

[8] Lemmens P., thesis, RWTH Aachen (1992).

[9] Palstra T.T.M., Batlogg B., Schneemeyer L.F., and Waszczak J.V., Phys. Rev. Lett. 61 (1988) 1662.

[10] Brandt E.H., Z. Phys. B 80 (1990) 167. 